TRANSACTIONS OF THE

AMERICAN MATHEMATICAL SOCIETY

Volume 350, Number 11, November 1998, Pages 4445-4459

S 0002-9947(98)01880-7

\title{
A $q$-DEFORMATION OF A TRIVIAL SYMMETRIC GROUP ACTION
}

\author{
PHIL HANLON AND RICHARD P. STANLEY
}

\begin{abstract}
Let $\mathcal{A}$ be the arrangement of hyperplanes consisting of the reflecting hyperplanes for the root system $A_{n-1}$. Let $B=B(q)$ be the Varchenko matrix for this arrangement with all hyperplane parameters equal to $q$. We show that $B$ is the matrix with rows and columns indexed by permutations with $\sigma, \tau$ entry equal to $q^{i\left(\sigma \tau^{-1}\right)}$ where $i(\pi)$ is the number of inversions of $\pi$. Equivalently $B$ is the matrix for left multiplication on $\mathbb{C S}_{n}$ by

$$
\Gamma_{n}(q)=\sum_{\pi \in \mathfrak{S}_{n}} q^{i(\pi)} \pi
$$

Clearly $B$ commutes with the right-regular action of $\mathfrak{S}_{n}$ on $\mathbb{C S}_{n}$. A general theorem of Varchenko applied in this special case shows that $B$ is singular exactly when $q$ is a $j(j-1)^{s t}$ root of 1 for some $j$ between 2 and $n$.

In this paper we prove two results which partially solve the problem (originally posed by Varchenko) of describing the $\mathfrak{S}_{n}$-module structure of the nullspace of $B$ in the case that $B$ is singular. Our first result is that

$$
\operatorname{ker}(B)=\operatorname{ind}_{\mathfrak{S}_{n-1}}^{\mathfrak{S}_{n}}\left(\operatorname{Lie}_{n-1}\right) / \operatorname{Lie}_{n}
$$

in the case that $q=e^{2 \pi i / n(n-1)}$ where $\operatorname{Lie}_{n}$ denotes the multilinear part of the free Lie algebra with $n$ generators. Our second result gives an elegant formula for the determinant of $B$ restricted to the virtual $\mathfrak{S}_{n}$-module $P^{\eta}$ with characteristic the power sum symmetric function $p_{\eta}(x)$.
\end{abstract}

\section{INTRODUCTION}

Let $\mathcal{A}=\left\{H_{1}, \ldots, H_{\ell}\right\}$ be an arrangement of hyperplanes in $\mathbb{R}^{n}$, let $\Delta$ be the union of the hyperplanes in $\mathcal{A}$ and let $\mathcal{R}$ be the set of regions in $\mathbb{R}^{n} \backslash \Delta$. In [V], Varchenko defines a matrix $B$ with rows and columns indexed by the regions in $\mathcal{R}$. This matrix depends on a collection of real parameters $a_{H}$, one for each hyperplane $H$ in $\mathcal{A}$. The $(R, S)$-entry of $B$ is given by

$$
B_{R, S}=\prod_{H} a_{H},
$$

where the product on the right is over hyperplanes $H$ which separate $R$ and $S$.

The matrices $B$ defined above are studied in papers of Varchenko [V], Schechtman/Varchenko $[\mathrm{SV}]$ and Brylawski/Varchenko [BV]. These matrices describe the

Received by the editors June 25, 1996 .

1991 Mathematics Subject Classification. Primary 20C30, 05E10.

The first author was partially supported by the National Science Foundation, Grant No. DMS9500979.

The second author was partially supported by the National Science Foundation, Grant No. DMS-9206374. 
analogue of Serre's relations for quantum Kac-Moody Lie algebras and are relevant to the study of hypergeometric functions and the representation theory of quantum groups. In these applications, the kernel of $B$ is of particular interest.

The kernel of $B$ depends on the values of the $a_{H}$. In general, the exact relationship is difficult to describe. But the following theorem of Varchenko gives considerable information about this dependence.

Theorem 1.1 (Varchenko $[\mathrm{V}])$. Let notation be as above. Then

$$
\operatorname{det}(B)=\prod_{F}\left(1-a(F)^{2}\right)^{p(F) q(F)},
$$

where the product is over the non-zero flats in the intersection lattice of $\mathcal{A}($ see $[\mathrm{O}])$, where $a(F)$ denotes the product of the $a_{H}$ over hyperplanes $H \in \mathcal{A}$ which contain $F$ and where $p(F)$ and $q(F)$ are non-negative integers defined in $[\mathrm{V}]$.

Our work in this paper will concern a particular arrangement. Fix a positive integer $n$. For each $i<j$ let $H_{i j}$ denote the hyperplane in $\mathbb{R}^{n}$ consisting of all $\left(x_{1}, \ldots, x_{n}\right)$ such that $x_{i}=x_{j}$. Let $\mathcal{A}_{n}$ be the arrangement consisting of all $H_{i j}$ with $1 \leq i<j \leq n$. For each $H \in \mathcal{A}_{n}$, let $a_{H}=q$ where $q$ is a complex indeterminate.

In this case $\Delta$ is the set of all vectors $\left(x_{1}, \ldots, x_{n}\right) \in \mathbb{R}^{n}$ which have (at least) one pair of equal coordinates. The regions in $\mathcal{R}$ are naturally indexed by permutations $\sigma$ in $\mathfrak{S}_{n}$ according to

(*) $\quad \sigma \in \mathfrak{S}_{n}$ indexes the region $R_{\sigma}=\left\{\left(x_{1}, \ldots, x_{n}\right): x_{\sigma 1}<x_{\sigma 2}<\cdots<x_{\sigma n}\right\}$.

Via this indexing, we can think of the rows and columns of $B$ as corresponding to permutations. We will let $B_{\sigma, \tau}$ denote the $\left(R_{\sigma}, R_{\tau}\right)$-entry of $B$ for $\sigma, \tau \in \mathfrak{S}_{n}$. In this way, we will think of $B$ as an endomorphism of $\mathbb{C S}_{n}$.

Lemma 1.2. Let $\Gamma_{n}(q) \in \mathbb{C}[q] \mathfrak{S}_{n}$ be defined by

$$
\Gamma_{n}(q)=\sum_{\sigma \in \mathfrak{S}_{n}} q^{i(\sigma)} \sigma
$$

where $i(\sigma)$ is the number of inversions of $\sigma$. Then $B$, considered as an endomorphism of $\mathbb{C S}_{n}$, is the matrix for left multiplication by $\Gamma_{n}(q)$.

Proof. Consider the $(\alpha, \beta)$-entry of $B$ for $\alpha, \beta \in \mathfrak{S}_{n}$. This entry is $q^{\ell}$ where $\ell$ is the number of hyperplanes $H_{j k}$ which must be crossed in going from $R_{\alpha}$ to $R_{\beta}$.

Note that:

$H_{j k}$ must be crossed in going from $R_{\alpha}$ to $R_{\beta}$,

iff: $\beta j>\beta k$ and $\alpha j<\alpha k$,

iff: $\beta j>\beta k$ and $\left(\alpha \beta^{-1}\right) \beta j<\left(\alpha \beta^{-1}\right) \beta k$,

iff: $(\beta j, \beta k)$ is an inversion of $\alpha \beta^{-1}$.

So the $(\alpha, \beta)$-entry of $B$ is equal to $q^{i\left(\alpha \beta^{-1}\right)}$ which proves the lemma.

Next we consider what Theorem 1.1 tells us in this case. The intersection lattice of $\mathcal{A}$ is the partition lattice $\Pi_{n}$. It turns out that the exponent $p(F)$ is 0 unless the partition $F$ has exactly one non-trivial block. If $F$ has one non-trivial block of size $\ell$, then $p(F)=(\ell-2) !(n-\ell+1)$ !. So in this case, Theorem 1.1 states that

$$
\operatorname{det}(B)=\prod_{\ell=2}^{n}\left(1-q^{\ell(\ell-1)}\right)^{\left(\begin{array}{l}
n \\
\ell
\end{array}\right)(\ell-2) !(n-\ell+1) !} .
$$


It should be noted that (1.1) was first proved by Zagier [Z]. In this paper we realize it as a special case of the Varchenko determinant factorization.

There is one other feature of this arrangement which makes it particularly interesting. The symmetric group acts on the set of regions $R_{\sigma}$ via the right-regular representation on the index. More precisely, if $\tau \in \mathfrak{S}_{n}$ then $\tau\left(R_{\sigma}\right)=R_{\sigma \tau}$. By Lemma 1.2, this action of $\mathfrak{S}_{n}$ commutes with $B$ and so $B$ preserves the isotypic components of this $\mathfrak{S}_{n}$-action and $\mathfrak{S}_{n}$ acts on the kernel of $B$.

Two questions motivated the work in this paper.

Question 1: What is the $\mathfrak{S}_{n}$-module structure of $\operatorname{ker}(B)$ at values of $q$ for which $\operatorname{det}(B)=0$ ?

Question 2: For each irreducible representation $S^{\lambda}$ of $\mathfrak{S}_{n}$, what is the determinant of the restriction of $B$ to the $S^{\lambda}$-isotypic component of $R$ ?

Question 2 was posed independently by Varchenko $[\mathrm{V}]$ and by Zagier $[\mathrm{Z}$, page 201]. Zagier's work concerns the relevance of the operator $B$ to a model in infinite statistics. This model, suggested by Hegstrom gives a $q$-deformation of the classical Bose $(q=1)$ and Fermi $(q=-1)$ statistics. It was studied for $q=0$ by Greenberg $[\mathrm{Gr}]$ and for general $q$ by Polyakov and Biedenharn [B].

In this paper we will prove a conjecture due to R. Stanley which answers a question which is equivalent to Question 2. With regard to Question 1, we will state a conjecture due to Hanlon and Varchenko which provides an elegant answer to Question 1 for a number of interesting values of $q$. We will prove this conjecture in one particularly interesting special case.

\section{A factorization of $\Gamma_{n}(q)$}

In this section we state a remarkable result which was first proved by Zagier [Z] and later re-discovered in the paper $[\mathrm{DKKT}]$. To state this result we need some notation.

We will use two embeddings of $\mathbb{C S}_{n-1}$ in $\mathbb{C S}_{n}$. The first is the usual embedding, namely we think of $\mathfrak{S}_{n-1}$ as the collection of permutations fixing $n$. More precisely, if $\sigma$ is in $\mathfrak{S}_{n-1}$, its image under this embedding is obtained from $\sigma$ by adding a one-cycle containing the number $n$. If $A \in \mathbb{C S}_{n-1}$ we let $A$ also denote its image in $\mathbb{C S}_{n}$ under this embedding.

The second embedding, which we denote $\eta$, maps $\mathfrak{S}_{n-1}$ to the collection of permutations in $\mathfrak{S}_{n}$ which fix 1 . If $\sigma \in \mathfrak{S}_{n-1}$ is written in disjoint cycle form then $\eta(\sigma)$ is obtained from $\sigma$ by increasing every number by 1 and then adding a onecycle containing the number 1 . For example, $\eta((1,3,5)(2,4))=(2,4,6)(3,5)(1)$. This defines $\eta$ on $\mathfrak{S}_{n-1}$ and it extends linearly to an embedding of $\mathbb{C S}_{n-1}$ into $\mathbb{C S}_{n}$.

For each $n$, let $T_{n}(q)$ denote the sum

$$
T_{n}(q)=\sum_{j=1}^{n} q^{j-1} \eta^{n-j}\left(z_{j}\right) .
$$

Here $z_{j}$ is the $j$-cycle $(j, j-1, \ldots, 1)$ in $\mathfrak{S}_{j}$ so $T_{n}(q)$, with permutations written out in disjoint cycle form, is

$$
T_{n}(q)=\sum_{j=1}^{n} q^{j-1}(n, n-1, \ldots, n-j+1) .
$$


It is straightforward to verify that

$$
\Gamma_{n}(q)=T_{2}(q) T_{3}(q) \cdots T_{n}(q) .
$$

The factorization result in [Z] gives a factorization of $T_{n}(q)$ which in turn gives a factorization of $\Gamma_{n}(q)$ by $(2.1)$.

To state the result, we need to define elements $G_{j}(q), H_{j}(q) \in \mathbb{C S}_{j}$ which we do inductively according to the following equations:

$$
\begin{aligned}
& G_{1}(q)=H_{1}(q)=1, \\
& G_{j}(q)=\left(1-q^{j} z_{j-1}\right) \eta\left(G_{j-1}(q)\right), \\
& H_{j}(q)=\eta\left(H_{j-1}(q)\right)\left(1-q^{j-1} z_{j}\right)^{-1} .
\end{aligned}
$$

Note that $H_{j}(q)$ is defined only for those values of $q$ with the property that $1-q^{\ell-1} z_{\ell}$ is invertible in $\mathbb{C S}_{j}$ for all $\ell \in\{2,3, \ldots, j\}$. It is straightforward to see that $1-q^{\ell-1} z_{\ell}$ is invertible in $\mathfrak{S}_{j}$ if and only if $1-q^{\ell(\ell-1)}$ is nonzero.

Theorem 2.1 ([Z, Prop. 2]). Suppose $\prod_{\ell=2}^{n}\left(1-q^{\ell(\ell-1)}\right)$ is nonzero. Then $T_{n}(q)$ $=G_{n}(q) H_{n}(q)$, and so

$$
\Gamma_{n}(q)=G_{2}(q) H_{2}(q) G_{3}(q) H_{3}(q) \cdots G_{n}(q) H_{n}(q) .
$$

This result can be extended to all values of $q$ by restating it as

$$
T_{n}(q)\left(1-q^{n-1} z_{n}\right)=\left(1-q^{n} z_{n-1}\right) \eta\left(T_{n-1}(q)\right) .
$$

As an example of (2.2), let $n=3$. Then (2.2) is the equation

$$
\begin{aligned}
(1+q(3,2) & \left.+q^{2}(3,2,1)\right)\left(1-q^{2}(3,2,1)\right) \\
& =\left(1-q^{3}(2,1)\right)(1+q(3,2)) .
\end{aligned}
$$

which is easily verified.

\section{The Main Results}

In this section we state and prove our two main results. We begin with a conjecture that is relevant to Question 1. In general, it seems that the $\mathfrak{S}_{n}$-module structure of the kernel of $\Gamma_{n}(q)$ is quite complicated. Below we see a table which gives the character values of $\mathfrak{S}_{n}$ acting on the kernel of $\Gamma_{4}(q)$ for all values for which $\Gamma_{4}(q)$ is singular. In this table the rows are indexed by values of $q$ and the columns by conjugacy classes in $\mathfrak{S}_{4}$ (denoted by cycle type).

\begin{tabular}{c|c|c|c|c|c} 
& $1^{4}$ & $21^{2}$ & $2^{2}$ & 31 & 4 \\
\hline 1 & 9 & -1 & 1 & 0 & -1 \\
-1 & 9 & 1 & 1 & 0 & 1 \\
$e^{2 \pi i / 3}$ & 3 & 1 & -1 & 0 & 1 \\
$e^{2 \pi i / 4}$ & 2 & 0 & 2 & 2 & 0 \\
$e^{2 \pi i / 6}$ & 3 & -1 & -1 & 0 & 1 \\
$e^{2 \pi i / 12}$ & 2 & 0 & 2 & -1 & 0
\end{tabular}

The reader will note that it is difficult to pick out a pattern even in the degrees of the representations. This observation is reinforced when one examines the same data for other values of $n$. However, Conjecture 3.1 states that the $\mathfrak{S}_{n}$-module structure of $\operatorname{ker}\left(\Gamma_{n}(q)\right)$ has a very elegant description for certain values of $q$. 
Conjecture 3.1. Suppose that $q$ is a root of exactly one of the factors on the right-hand side of (1.1). More precisely, suppose that $q=e^{2 \pi i s / j(j-1)}$ for some $j \in\{2,3, \ldots, n\}$ and some nonnegative integer $s$ and that $q^{k(k-1)} \neq 1$ for $k \neq$ $j, 2 \leq k \leq n$. Then

(a) The right $\mathfrak{S}_{n}$-module structure of $\operatorname{ker}\left(T_{n}(q)\right)$ is

$$
\operatorname{ker}\left(T_{n}(q)\right)=\operatorname{ind}_{C_{j-1}}^{\mathfrak{S}_{n}}\left(q^{j}\right) / \operatorname{ind}_{C_{j}}^{\mathfrak{S}_{n}}\left(q^{j-1}\right)
$$

where $C_{j-1}$ is the subgroup of $\mathfrak{S}_{n}$ generated by the $(j-1)$-cycle $z_{j-1}=(j-1, j-$ $2, \ldots, 2,1)$, and where $q^{j}$ denotes the linear character of $C_{j-1}$ whose value on $z_{j-1}$ is $q^{j}$.

(b) As a right $\mathfrak{S}_{n}$-module $\operatorname{ker}\left(\Gamma_{n}(q)\right)$ consists of $n-j+1$ copies of $\operatorname{ker}\left(T_{n}(q)\right)$

It is interesting to note that there are examples of $j(j-1)^{s t}$ roots of unity $q$ (with $2 \leq j \leq n$ ) for which $\operatorname{ind}_{C_{j}}^{\mathfrak{S}_{n}}\left(q^{j-1}\right)$ is not even contained in $\operatorname{ind}_{C_{j-1}}^{\mathfrak{S}_{n}}\left(q^{j}\right)$. If Conjecture 3.1 is correct, then this containment must hold whenever $1-q^{k(k-1)}$ is nonzero for all $k$ not equal to $j$.

We will prove Conjecture 3.1 in what is arguably the most interesting case. We denote by $\mathrm{Lie}_{n}$ the representation of $\mathfrak{S}_{n}$ on the multilinear part of the free Lie algebra with $n$ generators. There is an extensive literature on this representation (see for example $[\mathrm{G}]$, [R1], [R2]). We will need one fact about the $\mathrm{Lie}_{n}$ representation.

Lemma 3.2 ([R1, see p. 215]). For each $n$,

$$
\operatorname{Lie}_{n}=\operatorname{ind}_{C_{n}}^{\mathfrak{S}_{n}}\left(e^{2 \pi i / n}\right)
$$

Theorem 3.3. Let $q=e^{2 \pi i / n(n-1)}$. Then

$$
\operatorname{ker}\left(\Gamma_{n}(q)\right)=\operatorname{ind}_{\mathfrak{S}_{n-1}}^{\mathfrak{S}_{n}}\left(\operatorname{Lie}_{n-1}\right) / \operatorname{Lie}_{n}
$$

Before going ahead with the proof of Theorem 3.3 we will need a pair of technical lemmas.

Lemma 3.4. Suppose $j \leq n$. As an endomorphism of $\mathbb{C S}_{n}$ (acting by left multiplication) $T_{j}(q)$ has determinant

$$
\prod_{\ell=2}^{j}\left(1-q^{\ell(\ell-1)}\right)^{n ! / \ell(\ell-1)} .
$$

Proof. The proof is by induction on $n$. The result is easy to check for $n=1$ so assume the result is true for $n-1$.

First, suppose $j \leq n-1$. As an endomorphism of $\mathbb{C S}_{n-1}, T_{j}(q)$ has determinant

$$
\prod_{\ell=2}^{j}\left(1-q^{\ell(\ell-1)}\right)^{(n-1) ! / \ell(\ell-1)} .
$$

For each $i=1,2, \ldots, n$, let $V_{i}$ be the subspace of $\mathbb{C S}_{n}$ spanned by all permutations which map $i$ to $n$. Then $T_{j}(q)$ preserves each $V_{i}$ and the action of $T_{j}(q)$ on $V_{i}$ is clearly isomorphic to the action of $T_{j}(q)$ on $\mathbb{C S}_{n-1}$. It follows that the determinant of $T_{j}(q)$ on $\mathbb{C S}_{n-1}$ is the $n$th power of the quantity given in (3.1) which proves Lemma 3.4 in this case. 
Next suppose that $j=n$. By (2.1) we have

$$
\begin{aligned}
\operatorname{det}\left(T_{n}(q)\right) & =\operatorname{det}\left(\Gamma_{n}(q)\right) \prod_{j=2}^{n-1}\left(\operatorname{det}\left(T_{j}(q)\right)\right)^{-1} \\
& =\prod_{\ell=2}^{n-1}\left(1-q^{\ell(\ell-1)}\right)^{E_{\ell}}
\end{aligned}
$$

where

$$
\begin{aligned}
E_{\ell} & =\left(\begin{array}{l}
n \\
\ell
\end{array}\right)(\ell-2) !(n-\ell+1) !-(n-\ell)\left(\frac{n !}{\ell(\ell-1)}\right) \\
& =\frac{n !}{\ell(\ell-1)} .
\end{aligned}
$$

Here we have combined (1.1) with the cases $j<n$ for this lemma to get $E_{\ell}$. This completes the induction step.

The next lemma is a simple consequence of the Smith normal form.

Lemma 3.5. Let $M(z)$ be an $N$ by $N$ matrix whose entries are polynomials in $z$. Let $z_{0}$ be a complex number such that

$$
\operatorname{det}(M(z))=\left(z-z_{0}\right)^{D} f(z)
$$

where $f\left(z_{0}\right) \neq 0$. Then $\operatorname{dim}\left(\operatorname{ker}\left(M\left(z_{0}\right)\right)\right) \leq D$.

The third lemma is again a standard result from representation theory. Although it may not be explicitly stated in most references, it follows easily from the definition of induced representations.

Lemma 3.6. Let $H$ be a subgroup of a finite group $G$ and let $\psi$ be a linear character of $H$. Let $\tau$ be the element of $\mathbb{C} G$ given by:

$$
\tau=\frac{1}{|H|} \sum_{h \in H} \psi(h) h .
$$

Then the right ideal of $\mathbb{C} G$ generated by $\tau$ is isomorphic, as a right $G$-module, to $\operatorname{ind}_{H}^{G}(\psi)$.

We are now ready to proceed with the proof of Theorem 3.3. Let $q=e^{2 \pi i / n(n-1)}$. By Lemma 3.4, $T_{j}(q)$ is invertible as an endomorphism of $\mathbb{C S}_{n}$ for $j<n$. So $\operatorname{ker}\left(\Gamma_{n}(q)\right) \cong \operatorname{ker}\left(T_{n}(q)\right)$ where the isomorphism is one of right $\mathfrak{S}_{n}$-modules. Hence it is sufficient to show that

$$
\operatorname{ker}\left(T_{n}(q)\right) \cong \operatorname{ind}_{\mathfrak{S}_{n-1}}^{\mathfrak{S}_{n}}\left(\operatorname{Lie}_{n-1}\right) / \operatorname{Lie}_{n}
$$

Recall from $\S 2$ that

$$
T_{n}(q)\left(1-q^{n-1} z_{n}\right)=\left(1-q^{n} z_{n-1}\right) \eta\left(T_{n-1}(q)\right) .
$$

Our first step is to understand the kernel of the right-hand side of (2.2). Again appealing to Lemma 3.4 we have that $\eta\left(T_{n-1}(q)\right)$ is invertible as an endomorphism of $\mathbb{C S}_{n}$. So, as right $\mathfrak{S}_{n}$-modules,

$$
\operatorname{ker}\left(\left(1-q^{n} z_{n-1}\right) \eta\left(T_{n-1}(q)\right)\right) \cong \operatorname{ker}\left(1-q^{n} z_{n-1}\right) \text {. }
$$


It is straightforward to check that the kernel of $1-q^{n} z_{n-1}$ (acting as usual on $\mathbb{C S}_{n}$ by left-multiplication) is the right ideal generated by

$$
\tau=\frac{1}{n-1} \sum_{\ell=0}^{n-2}\left(q^{n} z_{n-1}\right)^{\ell} .
$$

So by Lemma 3.6, we have the following equalities of right $\mathfrak{S}_{n}$-modules:

$$
\operatorname{ker}\left(\left(1-q^{n} z_{n-1}\right)\right) \eta\left(T_{n-1}(q)\right)=\operatorname{ind}_{C_{n-1}}^{\mathfrak{S}_{n}}\left(q^{n}\right)=\operatorname{ind}_{\mathfrak{S}_{n-1}}^{\mathfrak{S}_{n}}\left(\operatorname{Lie}_{n-1}\right)
$$

(the last equality from Lemma 3.2).

We now consider the kernel of the left-hand side of (2.2). A similar argument involving Lemma 3.6 shows that

$$
\operatorname{ker}\left(1-q^{n-1} z_{n}\right) \cong \operatorname{ind}_{C_{n}}^{\mathfrak{S}_{n}}\left(q^{n-1}\right)=\operatorname{Lie}_{n},
$$

the last equality from Lemma 3.2. By standard linear algebra arguments,

$$
\operatorname{dim}\left(\operatorname{ker}\left(T_{n}(q)\left(1-q^{n-1} z_{n}\right)\right)\right) \leq \operatorname{dim}\left(\operatorname{ker}\left(T_{n}(q)\right)\right)+\operatorname{dim}\left(\operatorname{ker}\left(1-q^{n-1} z_{n}\right)\right)
$$

with equality if and only if

$$
\operatorname{ker}\left(T_{n}(q)\left(1-q^{n-1}\right)\right) \cong \operatorname{ker}\left(T_{n}(q)\right) \oplus \operatorname{ker}\left(\left(1-q^{n-1}\right)\right)
$$

(the last isomorphism being an isomorphism of right $\mathfrak{S}_{n}$-modules). Combining Lemmas 3.4 and 3.5 we have

$$
\operatorname{dim}\left(\operatorname{ker}\left(T_{n}(q)\right)\right) \leq(n-1) !
$$

This inequality together with (3.4) and (3.5) imply that

$$
\operatorname{dim}\left(\operatorname{ker}\left(T_{n}(q)\left(1-q^{n-1} z_{n}\right)\right)\right) \leq(n-2) !+(n-1) !=n(n-2) !
$$

with equality if and only if (3.6) holds. But by (3.3)

$$
\operatorname{ker}\left(T_{n}(q)\left(1-q^{n-1}\right)\right) \cong \operatorname{ind}_{\mathfrak{S}_{n-1}}^{\mathfrak{S}_{n}}\left(\operatorname{Lie}_{n-1}\right),
$$

which has dimension $n(n-2)$ !. This implies that there is equality in (3.7) and hence the isomorphism in (3.6) holds, i.e.,

$$
\operatorname{ind}_{\mathfrak{S}_{n-1}}^{\mathfrak{S}_{n}}\left(\operatorname{Lie}_{n-1}\right) \cong \operatorname{ker}\left(T_{n}(q)\right) \oplus \operatorname{Lie}_{n}
$$

It is interesting to note that the same representation

$$
\operatorname{ind}_{\mathfrak{S}_{n-1}}^{\mathfrak{S}_{n}}\left(\operatorname{Lie}_{n-1}\right) / \mathrm{Lie}_{n}
$$

has recently appeared in another quite different context. Let $\mathcal{F}_{n}$ denote the space of homeomorphically irreducible trees with $n$ labelled leaves. We think of $\mathcal{F}_{n}$ as a topological space with the trees near to each other if they differ by a small deformation (just as drawings in the plane). There is an action of $\mathfrak{S}_{n}$ on $\mathcal{F}_{n}$ induced by permutations of the labels on the leaves. This action lifts to an action of $\mathfrak{S}_{n}$ on $H_{*}\left(\mathcal{F}_{n}\right)$, the homology of $\mathcal{F}_{n}$. Sarah Whitehouse [W] showed that

$$
H_{*}\left(\mathcal{F}_{n}\right) \cong \operatorname{ind}_{\mathfrak{S}_{n-1}}^{\mathfrak{S}_{n}}\left(\mathrm{Lie}_{n-1}\right) / \mathrm{Lie}_{n}
$$

An interesting problem is to construct a $\mathfrak{S}_{n}$-equivariant isomorphism between $\operatorname{ker}\left(T_{n}(q)\right)$ and $H_{*}(\mathcal{F})$.

We next consider Question 2. As stated, this question probably does not have a nice answer. In Table 1 we see a chart which has rows indexed by partitions of 


\section{TABLE 1}

$\begin{array}{cl}\lambda & D_{S^{\lambda}}(B) \\ 1 & I_{0} \\ 2 & I_{2} \\ 1^{2} & I_{1} \\ 3 & I_{2} I_{3} \\ 21 & I_{1}^{6} I_{2}^{6} \\ 1^{3} & I_{1} I_{6} \\ & I_{2}^{2} I_{3} I_{4} \\ 4 & I_{1}^{18} I_{2}^{18} I_{3}^{9} \\ 2^{2} & I_{1}^{8} I_{2}^{8} I_{12}^{2} \\ 21^{2} & I_{1}^{18} I_{2}^{18} I_{6}^{9} \\ 1^{4} & I_{1}^{2} I_{4} I_{6} \\ 5 & I_{2}^{2} I_{3} I_{4} I_{5} \\ 41 & I_{1}^{10} I_{2}^{12} I_{3}^{6} I_{4}^{3} \\ 32 & I_{1}^{14} I_{2}^{14} I_{3}^{5} I_{10} I_{12}^{2} \\ 31^{2} & I_{1}^{16} I_{2}^{16} I_{3}^{5} I_{6}^{5} I_{20} \\ 2^{2} 1 & I_{1}^{14} I_{2}^{14} I_{5} I_{6}^{5} I_{12}^{2} \\ 21^{3} & I_{1}^{12} I_{2}^{10} I_{4}^{3} I_{6}^{6} \\ 1^{5} & I_{1}^{2} I_{4} I_{6} I_{10} \\ & I_{2}^{3} I_{3}^{2} I_{4} I_{5} I_{6} \\ 6 & I_{1}^{15} I_{2}^{18} I_{3}^{9} I_{4}^{6} I_{5}^{3} \\ 51 & I_{1}^{31} I_{2}^{34} I_{3}^{14} I_{4}^{5} I_{10}^{3} I_{12}^{3} I_{30} \\ 42 & I_{1}^{34} I_{2}^{36} I_{3}^{16} I_{4}^{5} I_{6}^{7} I_{15} I_{20} \\ 41^{2} & I_{1}^{18} I_{2}^{17} I_{3}^{8} I_{10} I_{12}^{3} I_{15} \\ 3^{2} & I_{1}^{5} I_{2}^{58} I_{3}^{15} I_{5}^{3} I_{6}^{115} I_{10}^{3} I_{12}^{6} I_{20}^{2} \\ 321 & I_{1}^{36} I_{2}^{34} I_{3}^{7} I_{4}^{5} I_{6}^{16} I_{20} I_{30} \\ 31^{3} & I_{1}^{17} I_{2}^{18} I_{5} I_{6}^{8} I_{12}^{3} I_{30} \\ 2^{3} & 2_{1}^{2} I_{2}^{21} I_{4}^{5} I_{5}^{3} I_{6}^{14} I_{12}^{3} I_{15} \\ 21^{4} & I_{1}^{18} I_{2}^{15} I_{4}^{6} I_{6}^{9} I_{10}^{3} \\ 1^{6} & I_{1}^{3} I_{3} I_{4} I_{6}^{2} I_{10} \\ & \\ & \end{array}$

small size. The entry in row $\lambda$ is the determinant $D_{S^{\lambda}}(B)$ of the restriction of $B$ to the $S^{\lambda}$-isotypic component of $\mathcal{R}$. In this chart we let $I_{j}$ denote the $j^{\text {th }}$ cyclotomic polynomial, i.e., the polynomial whose roots are the primitive $j^{\text {th }}$ roots of 1 .

To establish notation more generally, let $V$ be a virtual $\mathfrak{S}_{n}$-module written as

$$
V=\sum_{\lambda} n_{\lambda} S^{\lambda}
$$

where $n_{\lambda} \in \mathbb{Z}$. Let $A$ be an element of $\mathbb{C S}_{n}$. We let $D_{V}(A)$ denote the product over $\lambda$ of $\operatorname{det}\left(\varphi^{\lambda}(A)\right)^{n_{\lambda}}$ where $\varphi^{\lambda}$ is the irreducible representation of $\mathfrak{S}_{n}$ indexed by $\lambda$. 
TABLE 2

\begin{tabular}{rr}
$j$ & $d_{j}(T)$ \\
\hline 1 & 0 \\
2 & 0 \\
3 & 2 \\
4 & 2 \\
5 & 6 \\
6 & 11 \\
7 & 11 \\
8 & 18 \\
9 & 18
\end{tabular}

Next, for each $\eta \vdash n$, let $P^{\eta}$ denote the virtual representation

$$
P^{\eta}=\sum_{\lambda} \chi^{\lambda}(\eta) S^{\lambda}
$$

where $\chi^{\lambda}(\eta)$ is the value of the irreducible character $\chi^{\lambda}$ on the conjugacy class consisting of permutations of cycle type $\eta$. In terms of symmetric functions, $S^{\lambda}$ is the representation having the Schur function $s_{\lambda}(x)$ as characteristic whereas $P^{\eta}$ is the representation having the power sum symmetric function $p_{\eta}(x)$ as characteristic.

It is well-known that the character table $\left(\chi^{\lambda}(\eta)\right)$ of $\mathfrak{S}_{n}$ is an invertible matrix, so the determinants $D_{P^{\eta}}\left(T_{n}(q)\right)$ determine the determinants $D_{S^{\lambda}}\left(\Gamma_{n}(q)\right)$. The following conjecture of R. Stanley gives an elegant description of the $D_{P^{\eta}}\left(T_{n}(q)\right)$ 's.

Conjecture 3.7. For each $\eta \vdash n$, let $\mathcal{P}_{\eta}(q)$ denote $D_{P^{\eta}}\left(T_{n}(q)\right)$. Then

(a) $\mathcal{P}_{\eta}(q)=1$ unless $\eta$ is of the form $\ell^{d} 1^{n-\ell d}$ for some $\ell, d$.

(b) $\mathcal{P}_{\left(\ell^{d}, 1^{s}\right)}(q)=\left(\mathcal{P}_{\left(\ell^{d}, 1\right)}(q)\right)^{s !}$ for all $\ell, d$ and all $s \geq 1$.

Note that (a) and (b) reduce the determination of $\mathcal{P}_{\eta}(q)$ to the cases where $\eta$ is of the form $\ell^{d}$ or $\ell^{d} 1$.

(c) $\mathcal{P}_{\left(\ell^{d}\right)}(q)=\prod_{m \mid \ell}\left(1-q^{d m(n-1)}\right)^{\mu(m) \ell^{d}(d-1) ! / m}$

where in the exponent of the factors on the right-hand side, $\mu$ denotes the numbertheoretic Möbius function.

(d) $\mathcal{P}_{\left(\ell^{d}, 1\right)}(q)=\mathcal{P}_{\left(\ell^{d}\right)}(q) \mathcal{P}_{\left(\ell^{d}\right)}\left(q^{n /(n-2)}\right)^{-1}$.

The rest of this section will be devoted to proving Conjecture 3.7. We will need the following theorem which is a special case of a more general result proved by John Stembridge.

For $\lambda$ a partition of $n$, let $\mathcal{F}^{\lambda}$ denote the set of standard Young tableaux of shape $\lambda$. For $T \in \mathcal{F}^{\lambda}$ and $i \in\{1,2, \ldots, n-1\}$, we say $i$ is a descent of $T$ if $i+1$ occurs in a lower row of $T$ than $i$. For each $j$, let $d_{j}(T)$ denote the sum of the descents of $T$ which are less than $j$.

For example, if

$$
T=\begin{aligned}
& 12479 \\
& 358
\end{aligned}
$$

6

then the descents of $T$ are $2,4,5,7$. The numbers $d_{j}(T)$ are given in Table 2 . The special case of Stembridge's Theorem that we will need is: 
Theorem 3.8 (special case of Theorem 3.3 of [Ste]). Fix $j, \lambda$ with $1 \leq j \leq n$ and $\lambda \vdash n$. Let $\omega=e^{2 \pi i / j}$. Then the multiplicity of $\omega^{\ell}$ as an eigenvalue of $\varphi^{\lambda}\left(z_{j}\right)$ is equal to the number of $T \in \mathcal{F}^{\lambda}$ with $d_{j}(t) \equiv \ell(\bmod j)$.

Fix a positive integer $j$ and let $V$ be a $\mathfrak{S}_{n}$-module. Let $\omega=e^{2 \pi i / j}$. Following Stembridge we consider the generating function

$$
M_{V, j}(z)=\sum_{\ell} m_{V, j}\left(\omega^{\ell}\right) z^{\ell}
$$

where $m_{V, j}\left(\omega^{\ell}\right)$ is the multiplicity of $\omega^{\ell}$ as an eigenvalue of $z_{j}$ acting on $V$. For $V$, a virtual $\mathfrak{S}_{n}$-module written as $V=X-Y$ where $X$ and $Y$ are modules, define $M_{V, j}(z)$ to be $M_{X, j}(z)-M_{Y, j}(z)$. Theorem 3.8 above states

$$
M_{S^{\lambda}, j}(z)=\sum_{T \in \mathcal{F}^{\lambda}} z^{d_{j}(T)}
$$

where the sum on the right should be reduced modulo $1-z^{j}$. Another important result we will need is the following well-known result (see [M], pp. 49-50 for a proof).

Lemma 3.9. Let $\lambda$ be a partition of $n$. Then

$$
M_{S^{\lambda}, n}(z)=(z)_{n} s_{\lambda}\left(1, z, z^{2}, \ldots\right)
$$

where $(z)_{n}$ denotes $(1-z)\left(1-z^{2}\right) \ldots\left(1-z^{n}\right)$.

Lemma 3.10. Let $\eta, j$ be as above and let $\ell$ be a divisor of $j$.

(a) $M_{P^{\eta}, j}(z) \equiv(n-j) ! \sum_{s / \ell} \frac{1-z^{j}}{1-z^{s}} \mu\left(\frac{\ell}{s}\right) s \ell^{d-1}(d-1) !\left(\bmod 1-z^{j}\right)$ if $\eta=\ell^{d}, 1^{n-j}$, where d denotes $j / \ell$.

(b) $M_{P^{\eta}, j}(z) \equiv 0 \quad\left(\bmod 1-z^{j}\right)$ otherwise.

Proof.

$$
\begin{aligned}
M_{P^{\eta}, j}(z) & =\sum_{\lambda \vdash n} \chi^{\lambda}(\eta) M_{S^{\lambda}, j}(z) \\
& =\sum_{\lambda \vdash n} \chi^{\lambda}(\eta) \sum_{T \in \mathcal{F}^{\lambda}} z^{d_{j}(T)} .
\end{aligned}
$$

For $\alpha$ a partition of $j$ and $S \in \mathcal{F}^{\alpha}$, let $\mathcal{F}^{\lambda}(S)$ denote the subset of $\mathcal{F}^{\lambda}$ consisting of all $T$ which contain $S$. In other words, $T$ is contained in $\mathcal{F}^{\lambda}(S)$ if $S$ is obtained from $T$ by erasing the numbers $j+1, j+2, \ldots, n$. Note that if $T \in \mathcal{F}^{\lambda}(S)$ then $d_{j}(T)=d_{j}(S)$. Also, by the Littlewood-Richardson rule, $\left|\mathcal{F}^{\lambda}(S)\right|$ is equal to the multiplicity of $S^{\lambda}$ in the induction of $S^{\alpha}$ from $\mathfrak{S}_{j}$ to $\mathfrak{S}_{n}$,

$$
\left|\mathcal{F}^{\lambda}(S)\right|=\left\langle\chi^{\lambda}, \operatorname{ind}_{\mathfrak{S}_{j}}^{\mathfrak{S}_{n}}\left(\chi^{\alpha}\right)\right\rangle
$$

for every $S \in \mathcal{F}^{\alpha}$. It follows that

$$
M_{P^{\eta}, j}(z)=\sum_{\alpha \vdash n}\left(\sum_{S \in \mathcal{F}^{\alpha}} z^{d_{j}(S)}\right) \sum_{\lambda \vdash f}\left\langle\chi^{\lambda}, \operatorname{ind}_{\mathfrak{S}_{j}}^{\mathfrak{S}_{n}}\left(\chi^{\alpha}\right)\right\rangle \chi^{\lambda}(\eta) .
$$

Substituting the formula from Lemma 3.9 into equation (3.9) we obtain

$$
M_{P^{\eta}, j}(z)=(z)_{j} \sum_{\alpha \vdash j} s_{\alpha}\left(1, z, z^{2}, \ldots\right)\left(\operatorname{ind}_{\mathfrak{S}_{j}}^{\mathfrak{S}_{n}}\left(\chi^{\alpha}\right)\right)(\eta) .
$$


Note that $\left(\operatorname{ind}_{\mathfrak{S}_{j}}^{\mathfrak{S}_{n}}\left(\chi^{\alpha}\right)\right)(\eta)=0$ for all $\alpha$ unless $\eta$ is of the form $\eta=\bar{\eta} 1^{n-j}$ where $\bar{\eta} \vdash j$. So we have $M_{P^{\eta}, j}(z)=0$ in the case that $\eta$ is not of the form $\bar{\eta} 1^{n-j}$ for $\bar{\eta} \vdash j$ which proves Lemma 3.10 (b).

Suppose $\eta=\bar{\eta} 1^{n-j}$ where $\bar{\eta} \vdash j$. Then (see $[\mathrm{F}]$ )

$$
\left(\operatorname{ind}_{\mathfrak{S}_{j}}^{\mathfrak{S}_{n}}\left(\chi^{\alpha}\right)\right)(\eta)=\frac{n !|C(\bar{\eta})|}{j !|C(\eta)|} \chi^{\alpha}(\bar{\eta})
$$

where $C(\eta)$ is the conjugacy class in $\mathfrak{S}_{n}$ containing $\eta$ and $C(\bar{\eta})$ is the conjugacy class in $\mathfrak{S}_{j}$ containing $\bar{\eta}$. Thus

$$
\sum_{\alpha \vdash j} s_{\alpha}(\underline{x})\left(\operatorname{ind}_{\mathfrak{S}_{j}}^{\mathfrak{S}_{n}}\left(\chi^{\alpha}\right)\right)(\eta)=\frac{n !|C(\bar{\eta})|}{j !|C(\eta)|} \sum_{\alpha \vdash j} \chi^{\alpha}(\bar{\eta}) s_{\alpha}(\underline{x})=\frac{n !|C(\bar{\eta})|}{j !|C(\eta)|} p_{\bar{\eta}}(\underline{x}) .
$$

Substituting (3.11) in (3.10) we obtain

$$
M_{P^{\eta}, j}(z)=\frac{n !|C(\bar{\eta})|}{j !|C(\eta)|} \frac{(z)_{j}}{\Pi_{i}\left(1-z^{\bar{\eta}_{i}}\right)} .
$$

Note that $(z)_{j}=\left(1-z^{\ell}\right)^{j / \ell} u(z)$ where $u(z)$ is a polynomial in $z$. Hence $M_{P^{\eta}, j}(z) \equiv$ $0 \bmod 1-z^{\ell}$ unless $\bar{\eta}=\ell^{j / \ell}$. This observation completes the proof of Lemma 3.10 (b).

Lastly, assume that $\eta=\ell^{j / \ell}, 1^{n-j}$. Then

$$
|C(\eta)|=\left(\begin{array}{c}
n \\
\ell, \ell, \ldots, \ell, n-j
\end{array}\right) \frac{1}{(j / \ell) !}(\ell-1) !^{\mid j / \ell}
$$

and

$$
|C(\bar{\eta})|=\left(\begin{array}{c}
j \\
\ell, \ell, \ldots, \ell
\end{array}\right) \frac{1}{(j / \ell) !}(\ell-1) !^{j / \ell} .
$$

So

$$
\frac{n !|C(\bar{\eta})|}{j !|C(\eta)|}=(n-j) !
$$

Also,

$$
\frac{(z)_{j}}{\Pi_{i}\left(1-z^{\bar{\eta}_{i}}\right)}=\frac{(1-z)\left(1-z^{2}\right) \cdots\left(1-z^{j}\right)}{\left(1-z^{\ell}\right)^{j / \ell}} .
$$

Lemma 3.11. Suppose $\ell$ divides $j$. Let d denote $j / \ell$. Then

$$
\frac{(1-z)\left(1-z^{2}\right) \cdots\left(1-z^{j}\right)}{\left(1-z^{\ell}\right)^{d}}=\sum_{s \mid \ell} \frac{1-z^{j}}{1-z^{s}} \mu\left(\frac{\ell}{s}\right) \ell^{d-1}(d-1) !
$$

modulo $\left(1-z^{j}\right)$.

Proof. It is enough to show that the two sides are equal whenever $z$ is set equal to a $j^{\text {th }}$ root of unity. There are three cases to consider.

Case 1: $z^{j}=1$ but $z^{\ell} \neq 1$.

Clearly, both sides of the equality in Lemma 3.11 are equal to 0 .

Case 2: $z$ is a primitive $a^{\text {th }}$ root of unity for some $a$ which properly divides $\ell$ (i.e., $a<\ell)$. 
The left-hand side of the equality can be written as

$$
\left(\prod_{u=0}^{d-1} \prod_{i=1}^{\ell-1}\left(1-z^{u \ell+i}\right)\right) b(z)
$$

where $b(z)$ is a polynomial, so the left-hand side is equal to 0 (because of the factor $\left.1-z^{a}\right)$.

The only non-zero summands on the right-hand side are those indexed by $s$ where $a \mid s$. Hence the right-hand side is equal to

$$
\sum_{a|s| \ell}\left(\frac{j}{s}\right) \mu\left(\frac{\ell}{s}\right) s \ell^{d-1}(d-1) ! .
$$

We've used the fact that $\lim _{z \rightarrow \omega} \frac{1-z^{j}}{1-z^{s}}=\frac{j}{s}$ for $\omega$ an $s^{\text {th }}$ root of unity. We can rewrite (3.13) as

$$
\left\{\sum_{a|s| \ell} \mu\left(\frac{\ell}{s}\right)\right\} j \ell^{d}(d-1) !=0,
$$

the last equality following because $a$ is a proper divisor of $\ell$ so the sum of $\mu(e)$ for $e \mid\left(\frac{\ell}{a}\right)$ is 0 . This completes Case 2 .

Case 3: $z$ is a primitive $\ell^{\text {th }}$ root of unity.

We can write the left-hand side of the equation in Lemma 3.11 as

$$
\begin{aligned}
& \prod_{u=0}^{d-1}\left(\prod_{i=1}^{\ell-1}\left(1-z^{u \ell+i}\right)\right)\left(\sum_{\ell=0}^{u} z^{\ell \ell}\right) \\
& =\prod_{u=0}^{d-1}\left(\prod_{i=1}^{\ell-1}\left(1-z^{i}\right)\right)(u+1) \\
& =d ! \ell^{d} .
\end{aligned}
$$

The first equality in (3.14) uses the fact that $1-z^{u \ell+i} \equiv 1-z^{i} \bmod \left(1-z^{\ell}\right)$ and the second equality follows from the fact that

$$
\prod_{i=1}^{\ell-1}\left(1-z^{i}\right)=\left(\frac{x^{\ell}-1}{x-1}\right)_{x=1}=\ell .
$$

The right-hand side in Lemma 3.11 is equal to

$$
\frac{1-z^{j}}{1-z^{\ell}} \ell \ell^{d-1}(d-1) !=\ell^{d} d !
$$

which completes the proof of Lemma 3.11 .

Lemma 3.10 follows immediately.

We are now ready to prove Conjecture 3.7. This will require a translation between the multiplicative determinant statements of Conjecture 3.7 and the additive trace formulas in Lemma 3.10. This translation is given in the following proposition which we leave to the reader. 
Proposition 3.12. Let $\eta$ and $j$ be as above. Write $M_{P^{\eta}, j}(z)$ as

$$
M_{P^{\eta}, j}(z)=\sum_{u=0}^{j-1} c_{u} z^{u}
$$

Then

$$
D_{P^{\eta}}\left(1-X z_{j}\right)=\prod_{u}\left(1-X \omega^{u}\right)^{c_{u}}
$$

where $\omega=e^{2 \pi i / j}$.

Proof of Conjecture 3.7. Recall that $D_{P^{\eta}}(\alpha)$ denotes the determinant of $\alpha$ on the $\mathfrak{S}_{n}$-module $P^{\eta}$ for $\alpha \in \mathbb{C S}_{n}$. By Theorem 2.1 we have

$$
D_{P^{\eta}}\left(T_{n}(q)\right)=\prod_{j=1}^{n} D_{P^{\eta}}\left(1-q^{j} z_{j-1}\right)\left(D_{P^{\eta}}\left(1-q^{j-1} z_{j}\right)\right)^{-1}
$$

Case 1: $\eta$ is not of the form $\ell^{d} 1^{n-\ell d}$.

By Lemma 3.10(b) and Proposition 3.12, $D_{P \eta}\left(1-X z_{s}\right)=1$ for all $X$ and $z_{s}$. So $D_{P^{\eta}}\left(T_{N}(q)\right)=1$ which proves Conjecture $3.7(\mathrm{a})$.

Case 2: $\eta=\ell^{d}$.

By Lemma 3.10(b) and Proposition 3.12, $D_{P^{\eta}}\left(1-X z_{s}\right)=1$ for all $X$ and all $s<n$. So

$$
D_{P^{\eta}}\left(T_{n}(q)\right)=D_{P^{\eta}}\left(1-q^{n-1} z_{n}\right)^{-1} .
$$

By Lemma 3.10 and Proposition 3.12 we have

$$
D_{P^{\eta}}\left(T_{n}(q)\right)=\prod_{s \mid \ell} \prod_{i=0}^{\frac{n}{s}-1}\left(1-\omega^{i} q^{n-1}\right)^{-\mu\left(\frac{\ell}{s}\right) s \ell^{d-1}(d-1) !}
$$

where $\omega$ is a primitive $\left(\frac{n}{s}\right)^{t h}$ root of 1 . Letting $m=\ell / s$ we can rewrite this as

$$
D_{P^{\eta}}\left(T_{n}(q)\right)=\prod_{m \mid \ell}\left(1-q^{m d(n-1)}\right)^{-\mu(m) \ell^{d}(d-1) ! / m},
$$

which proves Conjecture 3.7(c).

Case 3: $\eta=m \ell^{d} 1^{n-j}$ where $j=\ell d<n$.

By Lemma 3.10(b) and Proposition 3.12,

$$
D_{P^{\eta}}\left(T_{n}(q)\right)=D_{P^{\eta}}\left(1-q^{j+1} z_{j}\right) / D_{P^{\eta}}\left(1-q^{j-1} z_{j}\right)
$$

By the same kind of computation as in Case 2 we have

$$
D_{P^{\eta}}\left(T_{n}(q)\right)=\left[\prod_{s \mid \ell} \frac{1-q^{j(j+1) / s}}{1-q^{j(j-1) / s}}\right]^{s \mu\left(\frac{\ell}{s}\right) \ell^{d-1}(d-1) !(n-j) !} .
$$

Conjecture 3.7(b) follows immediately from (3.16) as the only dependence on $n$ is the factor of $(n-j)$ ! in the exponent.

So assume $j=n-1$. From (3.16) we have

$$
D_{P^{\eta}}\left(T_{n}(q)\right)=U / V
$$


where

$$
U=\prod_{s \mid \ell}\left(1-q^{(n-1) / s(n-2)}\right)^{-s \mu\left(\frac{\ell}{s}\right) \ell^{d-1}(d-1) !}=D_{P^{\bar{\eta}}}(q)
$$

and

$$
V=\prod_{s \mid \ell}\left(1-q^{((n-1) / s) n}\right)^{-s \mu\left(\frac{\ell}{s}\right) \ell^{d-1}(d-1) !}=D_{P_{\bar{\eta}}}\left(q^{n / n-2}\right)
$$

where $\bar{\eta}=\ell^{d}$. This proves Conjecture 3.7(d) and completes the proof of Conjecture 3.7 .

\section{Other Problems}

Let $\mathcal{A}$ be an arrangement of hyperplanes in $\mathbb{R}^{n}, G$ a finite group of linear transformations of $\mathbb{R}^{n}$, which acts on $\mathcal{A}$ and let $a_{H}$ be an assignment of parameters which is $G$-invariant. Then the Varchenko matrix $B$ commutes with $G$ and so we can ask the same questions in this more general setting that we addressed in this paper. More precisely we can ask:

1) For each choice of ( $G$-invariant) parameter weights giving $\operatorname{det}(B)=0$, what is the $G$-module structure of the kernel of $B$ ? Does this question have a simple answer in the case that the factors in Theorem 1.1 which are 0 correspond to only one orbit of flats in the intersection lattice of $\mathcal{A}$ ?

2) What is the determinant of the restriction of $B$ to each isotypic component of the $G$-action on regions?

A second line of inquiry suggested by the results in the paper is to answer questions 1) and 2) for the endomorphism $B$ of $\mathbb{C} G$ corresponding to left multiplication by

$$
\sum_{\sigma \in G} q^{\ell(\sigma)} \sigma
$$

for other Coxeter groups $G$, where $\ell$ is the length function in $G$.

In another direction, note that Conjecture 3.1 (b) implies that the dimension of the kernel of $\Gamma_{n}(q)$ is equal to the exponent of the $j^{\text {th }}$ cyclotomic polynomial in $\operatorname{det}\left(\Gamma_{n}(q)\right)$ in the case that $q$ is a primitive $j^{\text {th }}$ root of unity which divides exactly one of the factors in (1.1). This is equivalent to saying that the $j^{\text {th }}$ cyclotomic polynomial appears with exponent at most one in the diagonal terms of the Smith Normal Form of $\Gamma_{n}(q)$ (over the ring $\mathbb{C}[q]$ ). Based on computational evidence we make the following more general conjecture:

Conjecture 4.1. The maximum exponent of the $j^{\text {th }}$ cyclotomic polynomial $I_{j}(q)$ in a diagonal entry of the Smith Normal Form of $\Gamma_{n}(q)$ is equal to the number of factors in (1.1) that are divisible by $I_{j}(q)$.

It would be interesting to better understand the Smith Normal Forms of the Varchenko matrices over $\mathbb{C}[q]$ when all weights $a_{H}$ are set equal to $q$.

\section{REFERENCES}

[B] L.C. Biedenharn, J. Phys. A (1989), L873-L878. MR 90k:17027

[BV] T. Brylawski and A. Varchenko, "The determinant formula for a matroid bilinear form", preprint. 
[DKKT] G. Duchamp, A.A. Klyachko, D. Krob, J.Y. Thibon, "Noncommutative symmetric functions III: Deformations of Cauchy and convolution algebras", preprint.

[F] W. Feit, Characters of Finite Groups, Yale University Press, New Haven, Conn., 1965.

[G] A. Garsia, "Combinatorics of the free Lie algebra and the symmetric group", Analysis etcetera, J. Moser's festschrift, Academic Press, New York (1990). MR 91a:17006

[Gr] O.W. Greenberg, "Example of infinite statistics", Phys. Rev. Lett., 64 (1990), 705-708. MR 90k: 81080

[M] I.G. Macdonald, Symmetric Functions and Hall Polynomials, Oxford University Press, 1979. MR 84g:05001

[O] P. Orlik, Introduction to arrangements, CBMS Regional Series in Mathematics, \#72, AMS, Providence, RI (1989). MR 90i:32018

[R1] C. Reutenauer, Free Lie Algebras, Oxford University Press, 1993.

[R2] C. Reutenauer, "Theorem of Poincaré-Birkhoff-Witt and symmetric group representations of degrees equal to the Stirling numbers", Lecture Notes in Math., vol. 1234, Springer-Verlag, N.Y. (1986). MR 89i:05029

[Ro] A. Robinson, "The space of fully grown trees", preprint.

[Ste] J. Stembridge, "On the eigenvalues of representations of reflection groups and wreath products", Pacific J. Math., 140, No. 2 (1989), 353-396. MR 91a:20022

[SV] V. Schechtman and A. Varchenko, "Arrangements of hyperplanes and Lie algebra homology" Inventiones Math., 106 (1991), 139-194. MR 93b:17067

[V] A. Varchenko, "Bilinear form of real configuration of hyperplanes", Adv. in Math. 97 (1993), 110-144. MR 94b:52023

[W] S. Whitehouse, "Gamma homology of commutative algebras and some related representations of the symmetric group", preprint.

[Z] D. Zagier, "Realizability of a model in infinite statistics", Comm. in Math. Phys., 147 (1992), 199-210. MR 93i:81105

Department of Mathematics, University of Michigan, Ann Arbor, Michigan 481091109

E-mail address: phil@math.lda.umich.edu

Department of Mathematics 2-375, Massachusetts Institute of Technology, CamBRIDGe, Massachusetts 02139

E-mail address: rstan@math.mit.edu 